\title{
Comparison of Different Phenotypic Tests for the Detection of Metallo-B-Lactamase (MBL) Producing Pseudomonas aeruginosa
}

\author{
S. Soumya* and Mahantesh B. Nagmoti \\ Jawaharlal Nehru Medical College, KLE University, Belagavi, India \\ *Corresponding author
}

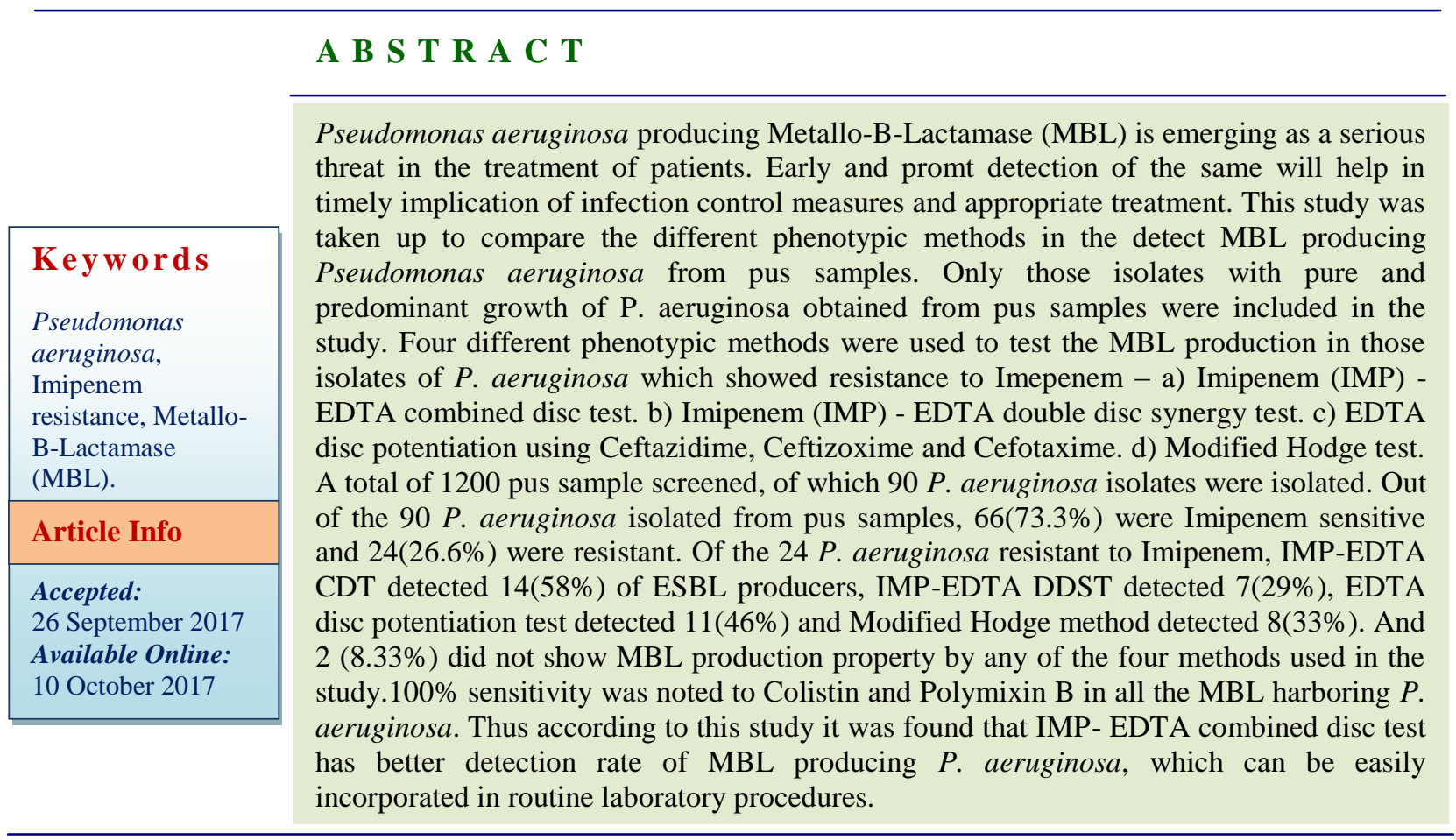

\section{Introduction}

Due to the wide use of $\beta$ - lactams especially the carbapenems in the treatment of betalactam resistant bacteria including ESBL producing bacteria, MBL producing organisms have increased in number and are posing a serious problem in treating them. ${ }^{1}$

MBL production has been increasingly reported among Pseudomonas aeruginosa, which is an important nosocomial multidrug resistant pathogen. ${ }^{2,3}$ Metallo beta lactamase (MBL) belongs to Ambler class B enzymes.
With the help of divalent cation zinc as cofactor for enzymatic activity, they hydrolyze carbapenem as-well-as other blactam antibiotics. The substrate spectrum of this enzyme is quite broad, as it can hydrolyze penicillins, cephalosporins and carbapenems, but it lacks the ability to hydrolyze aztreonam. ${ }^{4}$ Genes responsible for MBL production are IMP and VIM and they are coded by plasmids which in turn are involved in transfer of resistance from one bacteria to another. ${ }^{5}$ Chromosomal MBL was first 
detected in environmental and opportunistic pathogenic bacteria such as Bacillus cereus, Aeromonas spp., and Stenotrophomonas maltophilia. Lately, there has been a dramatic increase in the detection and spread of acquired and transferable families of these metallo- enzymes (IMP, VIM, SPM, GIM, SIM and AIM enzymes). ${ }^{4}$ In 1991, Japan reported the first case of Pseudomonas aeruginosa producing and from then it has been reported in various parts worldwide. ${ }^{6}$

CLSI guidelines don't mention about the standard available and reliable method for detection of these enzymes in P. aeruginosa, ${ }^{7}$

This study was taken up, to compare different phenotypic tests for identification of producing $P$. aeruginosa from pus samples.

This possibly will help to prevent the associated morbidity and mortality caused due to this organism by implementing proper control measures and to evaluate antibiotic therapy.

\section{Materials and Methods}

A total of 1200 pus samples were screened in one year which were received at the Department of Microbiology, J. N. Medical College, KLE university, from hospitalized patients of K.L.E.'S DR. Prabhakar Kore's Charitable Hospital and MRC, Belagavi were processed.

Isolates of $P$. aeruginosa obtained from pus samples as pure and predominant growth were considered for the study. Based on colony morphology and biochemical tests, organisms were identified. Using disc diffusion method, sensitivity of the isolates to imepenem/meropenem was determined using the control strain of $P$. aeruginosa ATCC 27853. CLSI guidelines were followed to interpret the results.
MBL production was tested only in those isolates of $P$. aeruginosa showing resistance to Imepenem, by the following methods

Imipenem (IMP) - EDTA combined disc test

Imipenem (IMP) - EDTA double disc synergy test

EDTA disc potentiation using ceftazidime, ceftizoxime and cefotaxime

Modified Hodge test.

Controls used For MBL tests ${ }^{8}$ :

Positive control-P. aeruginosa ATCC 68549

Negative control-P. aeruginosa ATCC 27853

\section{EDTA solution preparation}

$186.1 \mathrm{~g}$ of disodium EDTA. $2 \mathrm{H}_{2} \mathrm{O}$ was dissolved in $1000 \mathrm{ml}$ of distilled water. $\mathrm{pH} 8$ was adjusted using using Sodium hydroxide $(\mathrm{NaOH})$ and then autoclaving for sterilization. Each time $10 \mu \mathrm{l}$ of $0.5 \mathrm{M}$ EDTA solution was used for the test. Solution prepared can be stored in airtight vials for 16 weeks in refrigerator at $4^{0} \mathrm{C}$ or $-20^{0} \mathrm{C}$ without significant loss of activity. ${ }^{9}$

\section{MBL screening tests}

\section{Imipenem (IMP) - EDTA combined disc test $^{10}$}

Two 10mcg Imipenem discs are placed on the MHA plate which was prepared by lawn culture of standard inoculum (0.5 McFarland) of the test organism. To one of the IMP discs, $10 \mu \mathrm{l}$ of $0.5 \mathrm{M}$ EDTA solution was added to obtain a concentration of $750 \mathrm{mcg}$ and was incubated overnight at $37^{\circ} \mathrm{C}$.

Isolate was considered as MBL producer if $>7 \mathrm{~mm}$ increase in inhibition zone with IMP- 
EDTA disc compared to IMP disc alone was noted.

\section{Imipenem (IMP) - EDTA double disc synergy test ${ }^{10}$}

An IMP (10mcg) disc and a blank disc $(6 \mathrm{~mm}$ diameter, Whatmann No.2) containing $10 \mathrm{mcl}$ of $0.5 \mathrm{M}$ EDTA(750mcg) were placed $20 \mathrm{~mm}$ center to center on the MHA plate prepared with lawn culture of 0.5 McFarland suspension of the test organism and then was incubated overnight at $37^{\circ} \mathrm{C}$.

Isolate was considered as MBL producer if any increased zone of inhibition in the area between IMP and EDTA disc in comparison with zone of inhibition on far side of the drug was seen.

\section{EDTA disc potentiation using ceftazidime, ceftizoxime and cefotaxime ${ }^{10}$}

A black disc containing $10 \mathrm{mcl}$ of $0.5 \mathrm{M}$ EDTA (750mcg) solution was placed at the center of the plate and then the above three antibiotic discs were placed $25 \mathrm{~mm}$ center to center from blank disc on the MHA plate prepared with lawn culture of $0.5 \mathrm{McFarland}$ suspension of the test organism, followed by overnight incubation at $37^{\circ} \mathrm{C}$.

Isolate was considered as MBL producer if any increase in the inhibition zone in the area between EDTA disc and any one of the three cephalosporin disc in comparison with zone of inhibition on far side of the drug was seen.

\section{Modified Hodge test ${ }^{11}$}

MHA plate was lawn cultured with 0.5 McFarland of the E.coli ATCC 25922. Test organism is streaked across the plate to form a plus sign and the an IMP disc (10mcg) is placed at the center of the plate, followed byn overnight incubation at $37^{\circ} \mathrm{C}$.
Cloverleaf shaped inhibition is considered as a carbapenemases producer.

\section{Results and Discussion}

Out of 1200 pus sample screened, $90 P$. aeruginosa isolates were isolated.

Out of the $90 P$. aeruginosa isolated from pus samples, 66(73.3\%) were Imipenem sensitive and $24(26.6 \%)$ were resistant.

Of the $24 \quad P$. aeruginosa resistant to imipenem, IMP-EDTA CDT detected $14(58 \%)$ of ESBL producers, IMP-EDTA DDST detected 7(29\%), EDTA disc potentiation test detected 11(46\%) and Modified Hodge method detected 8(33\%). And $2(8.33 \%)$ did not show MBL production property by any of the four methods used in the study (Figs 1-3).

Pseudomonas aeruginosa exhibits intrinsic resistance to several antimicrobial agents, thus becoming the leading cause for various nosocomial infections. However, acquired resistance to anti-pseudomonal $\beta$-lactams such as Piperacillin, Aztreonam, Cefepime, Ceftazidime, and Carbapenems considered as deterrent weapon that can be a major challenge in managing MDRPA infections, especially while it is associated with coresistance with other classes of drugs namely Aminoglycosides and Fluoroquinolones.

Several mechanisms contributing to the acquired $\beta$-lactam resistance in $P$. aeruginosa. Few of them are-decreased outer membrane permeability, production of $\beta$-lactamases mainly MBL or the upregulation of efflux systems.

In the present study $26.6 \% \quad(24 / 90) \quad P$. aeruginosa were resistant to Imipenem which is similar to study done by Shobha et al., ${ }^{8}$, showing $30 \%$ of Imipenem resistant $P$. 
aeruginosa. Another study done by Varaiya $e t$ al., ${ }^{12}$, showed $20.8 \%$ P. aeruginosa resistant to Imipenem. A study by John $\mathrm{S}$ et al., ${ }^{13}$, showed $27.7 \%$, Zahra et al. ${ }^{14}$, showed $30.2 \%$, Irfan et al., ${ }^{15}$, showed $25 \%$ and Kumar et al., ${ }^{16}$, showed $32.4 \%$ Imipenem resistant $P$. aeruginosa.

Study in few other places like Bangalore, by Navneeth et al., found $12 \%$ of MBL producing $P$. aeruginosa. ${ }^{17}$ which is less in comparison to our study.

Variation in sample size studied or differences in hygienic practices may be the cause for the difference in the prevalence of MBL producing $P$. aeruginosa in different places/studies.

In the present study, IMP-EDTA CDT was a better method compared to other methods for detection of MBL producing $P$. aeruginosa, as in this study, of the 24 Imipenem resistant $P$. aeruginosa, Imipenem (IMP)- EDTA combined disc test detected 14(58\%) and EDTA disc potentiation using Ceftazidime, Ceftizoxime and Cefotaxime which detected 11(46\%), Modified Hodge test detected $8(33 \%)$ and Imipenem(IMP)- EDTA double disc synergy test detected 7(29\%).This is in accordance with a similar study by Behera $e t$ al., ${ }^{10}$, showed CDT is superior to DDST and DPT for detection on MBL producing $P$. aeruginosa.

Another study by Yan JJ et al., ${ }^{18}$ also showed that CDT is better method to detect MBL producing $P$. aeruginosa. Murugan et al., showed DDST detected $70 \%$ and MHT detected $50 \%$ of MBL producing $P$. aeruginosa and hence showed DDST was more accurate than MHT in detecting MBL producing $P$. aeruginosa. ${ }^{19}$

Pragapati SB et al., ${ }^{20}$ showed DDST detected $16(27.11 \%)$ and CDT detected $30(50.84 \%)$ of MBL producers, which is similar to a study done by Boghiel et al., ${ }^{21} \mathrm{MBL}$ producer prevalence study done by Agrawal G et in clinical isolates of Pseudomonas aeruginosa using DDST and MIC methods using 174 isolates gave same results for both the combinations, and showed that MIC reduction is a cumbersome, laborious method. Thus found DDST to be the performed method.

And hence, DDST is recommended for detection of MBL production in $P$. aeruginosa as a screening method. ${ }^{22}$

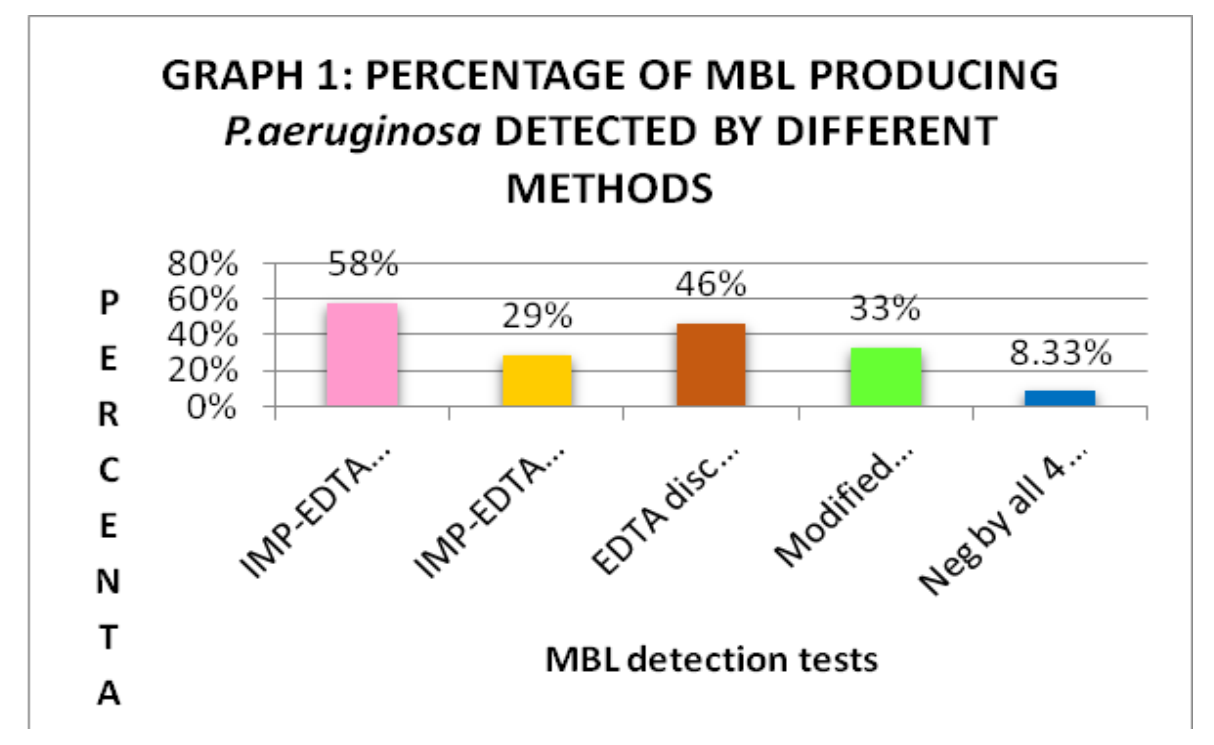


Fig.1 Imipenem (IMP)-EDTA combined disc test (IMP-EDTA CDT). Imipenem (IMP)-EDTA double disc synergy test (DDST)

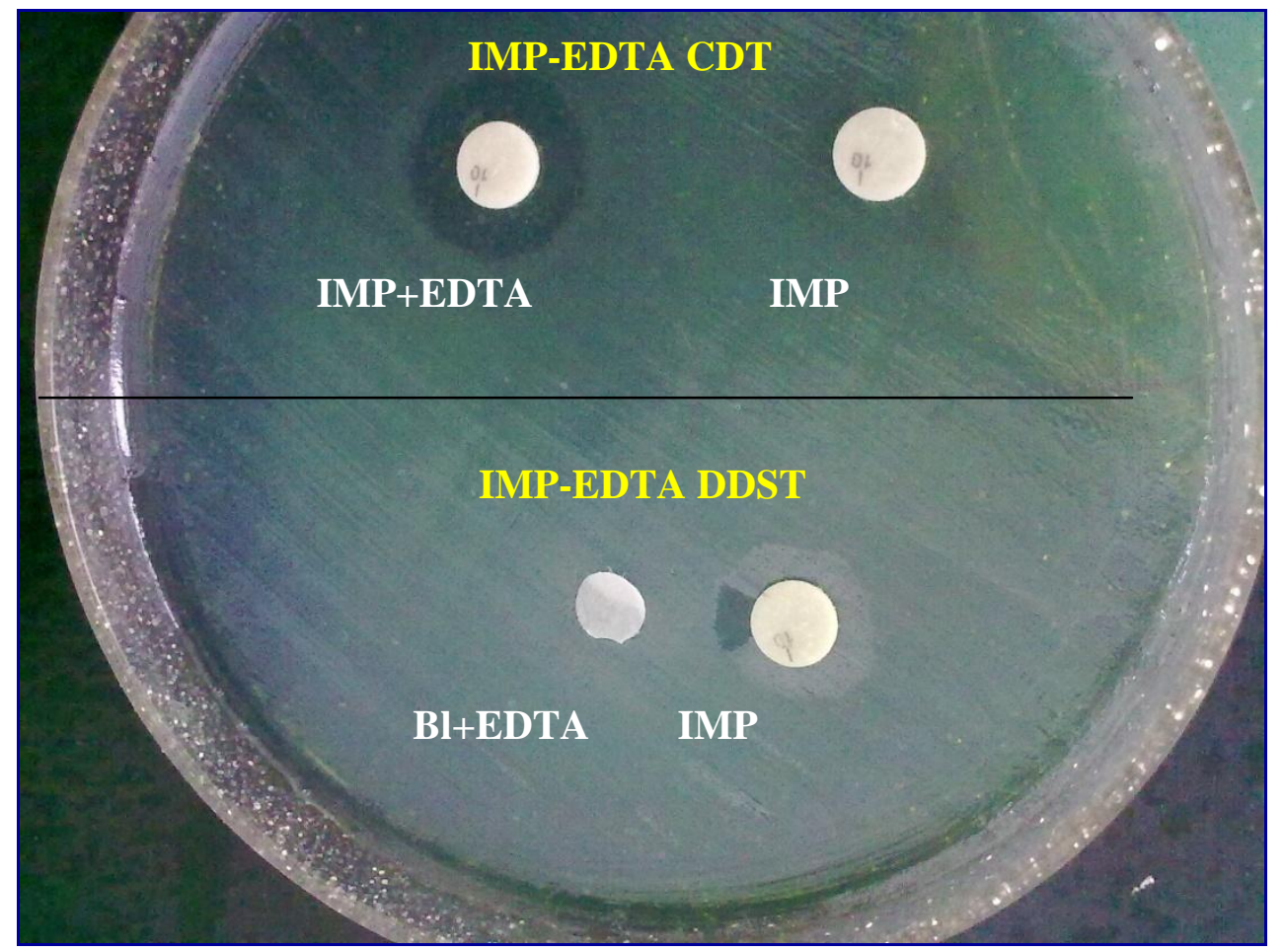

Fig.2 EDTA disc potentiation using Ceftazidime, Ceftizoxime, and Cefotaxime

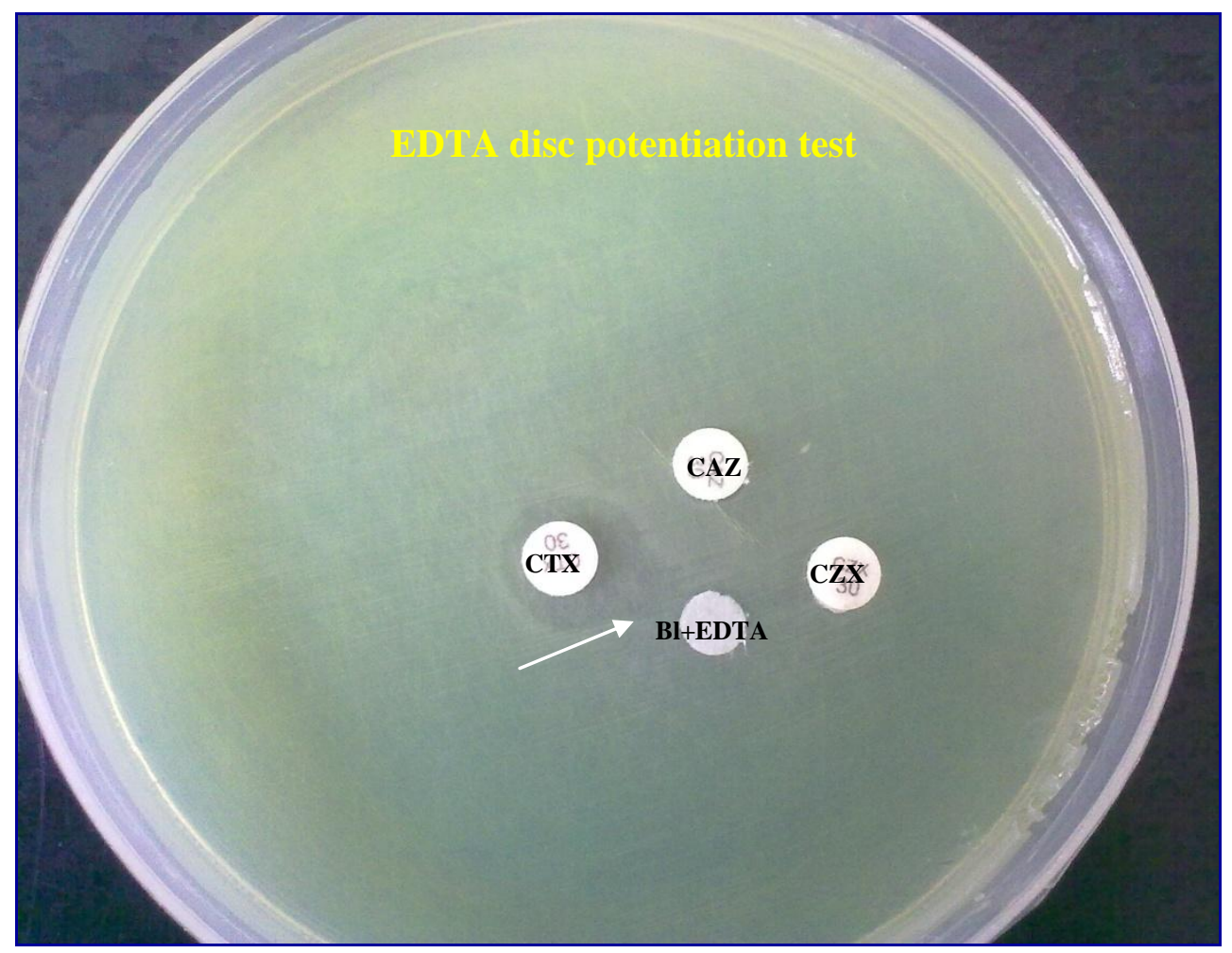


Fig.3 Modified Hodge test

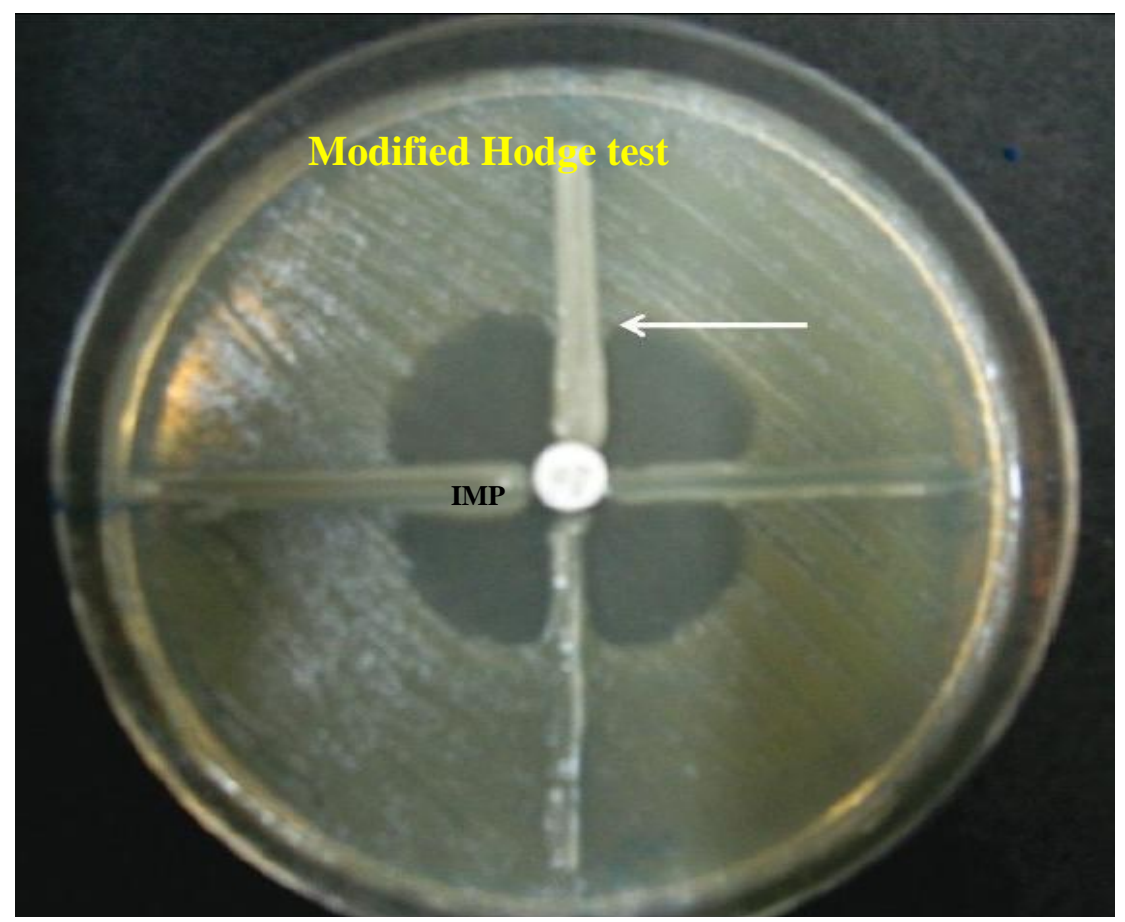

A study by Noyal et al., showed of the $18.9 \%$ Merapenem resistant, 16 (50.0\%) were MBL producers by EDTA disk synergy test and 9 (28.1\%) positive by modified Hodge test, and hence prepared method for MBL detection was eTDA disk synergy test over modified Hodge test. ${ }^{23}$

Impermeability of outer membrane and or active efflux mechanism could be the possible reasons for the two (8.33\%) Imepenim resistant $P$. aeruginosa which gave negative results for the detection of MBL producer by all the four methods used.

The main limitations of this study are, due to the absence of any standard methods to detect MBL in non-fermenters, it is difficult to comment on true or false MBL producers, MIC reduction would be a better method to know the drug susceptibility, but it is a cumbersome, laborious method and PCR could have been an additional investigation to detect the genes responsible for resistant, but has the disadvantage of its high cost.
Of the total 90 patients from whom $P$. aeruginosa were isolated, those which were not ESBL and MBL producers improved with treatment by third generation Cephalosporins, Erythromycin, Ampicillin and Ciprofloxacin ear drops. 3 patients were take-up for amputation, 3 of them expired due to development of septicemia and 12 patients could not be followed up.

All the patients in whom MBL producing $P$. aeruginosa were treated with colistin and polymixin B.

Thus according to this study it was found thatfor the detection of MBL producing $P$. aeruginosa Imipenem (IMP)- EDTA combined disc test is a best method.

\section{Interpretation of IMP-EDTA DDST}

Isolate is considered as $\mathrm{MBL}$ producer if $>7 \mathrm{~mm}$ increase inhibition zone size of with IMP-EDTA combined disccompared to IMP disc alone was noted. 


\section{Interpretation of IMP-EDTA CDT}

Isolate is considered as MBL producer if, any increase in inhibition zone of IMP in the area between IMP and blank disc + EDTA disc when comparison far side inhibition zone of IMP drugnoted.

\section{Interpretation of EDTA disc potentiation test}

Isolate is considered as MBL producer if, any increase in the inhition zone in the area between blank disc+EDTA disc and CTX in comparison far side of drug inhibition zone noted.

\section{Interpretation of Modified Hodge Test}

Cloverleaf shaped zone of inhibition around the IMP disc

Increased occurrence of MBL producing $P$. aeruginosa in clinical strains is alarming and reflects the excessive use of carbapenem by the clinicians. Thus early and accurate detection of MBL producing $P$. aeruginosa has helped the doctors to treat the patients early with appropriate antibiotics, thereby improving the patient outcome and decreased the morbidity and mortality. And it also calls for a strict need of following antibiotic policy to avoid excess and inappropriate use of carbapenems.

Thus according to this study it was found that Imipenem (IMP) - EDTA combined disc test to be the prefered method for detection of MBL producing $P$. aeruginosa.

\section{References}

Agrawal G, Lodhi RB, Kamalakar UP, Khadse RK, Jalgaonkar SV. Study of metallo-betalactamase production in clinical isolates of Pseudomonas aeruginosa. Indian $\mathrm{J}$ Med Microbiol.2008;26(4):349-51.
Amutha R, Padmakrishnan, Murugan $\mathrm{T}$ and Renugadevi M P. Study of multi drug resistant $P$. aeruginosa from pediatric population with special reference to ESBL. Indian J.Sci.Technol.2009;11(2):11-13.

Behera B, Mathur P, Das A, Kapil A, Sharma V. An evaluation of four different phenotypic techniques for detection of metallo- $\beta$ lactamase producing Pseudomonas aeruginosa. Indian $\mathrm{J}$ Med Microbiol. 2008;26:233-7.

Bush K, Jacoby GA, Medeiros AA. A functional classification scheme for b-lactamases and its correlation with molecular structure. Antimicrobial Agents Chemother. 1995;39:1211-33.

Gladstone P, Rajendran P, Brahmadathan KN. Incidence of carbapenem resistant nonfermenting Gram negative bacilli from patients with respiratory infections in the intensive care unit. Indian J Med Microbiol 2005; 23 : 189-91.

Irfan S, Zafar A, Guhar D, Ahsan T, Hasan R. Metallo-beta-lactamase-producing clinical isolates of Acinetobacter species and Pseudomonas aeruginosa from intensive care unit patients of a tertiary care hospital. Indian J Med Microbiol. 2008; 26(3):243-5.

John S, Balagurunathan R. Metallo beta lactamase producing Pseudomonas aeruginosa and Acinetobacter baumannii. Indian J Med Microbiol. 2011; 29(3):302-4.

Kumar SH, De AS, Baveja SM, Gore MA. Prevalence and Risk Factors of Metallo $\beta$ lactamase Producing Pseudomonas aeruginosa and Acinetobacter species in Burns and Surgical Wards in a Tertiary Care Hospital. J Lab Physicians.2012; 4(1):39-42.

Kumar SH, De AS, Baveja SM, Gore MA. Prevalence and Risk Factors of Metallo $\beta$ lactamase Producing Pseudomonas aeruginosa and Acinetobacter species in Burns and Surgical Wards in a Tertiary Care Hospital. J Lab Physicians.2012; 4(1):39-42.

Li J, Yang JY, Yan LN, Wang WT, Xu MQ, Wu H. Clinical distribution and antibiotic resistance of non-fermentative Gramnegative bacilli infection after liver 
transplantation. Sichuan Da Xue Xue Bao Yi Xue Ban. 2007;38(6):543-6.

Livermore DM. Multiple mechanisms of antimicrobial resistance in Pseudomonas aeruginosa: our worst nightmare? Clin Infect Dis. 2002; 34:634-40, doi: $10.1086 / 338782$.

Murrugan S, Laxmi RB, UmaDevi P and Mani KR.Prevalence and Antimicrobial Susceptibility Pattern Of Metallo $\beta$ lactamase Producing $P$. aeruginosa in Diabetic Foot Infection. Intl.J.Microbiol.Res.2010;1(3):123-128.

Murrugan S, Laxmi RB, UmaDevi $\mathrm{P}$ and Mani KR.Prevalence and Antimicrobial Suseptibility Pattern Of Metallo $\beta$ lactamase Producing $P$. aeruginosa in Diabetic Foot Infection .Intl.J.Microbiol.Res.2010;1(3):123-128.

Navaneeth BV, Sridaran D, Sahay D, Belwadi MR. A preliminary study on metallo-betalactamase producing Pseudomonas aeruginosa in hospitalized patients. Indian J Med Res. 2002;116(7):264-7.

Neu HC. The role of Pseudomonas aeruginosa in infections. J Antimicrob Chemother.1983;11 Suppl B:1-13.

Noyal MJC, Menezes GA, Harish BN, Sujatha S and Parija SC. Simple screening tests for detection of carbepenemases in clinical isolates of Non-fermentative gram-negative bacteria. Indian J Med res. 2009;129(6):707-712.

Prajapati S B, Vegad M M, Mehta S J, Kikani K $\mathrm{M}$, Kamothi $\mathrm{M} \mathrm{N}$ and Pandya J M.An evaluation of two different phenotypic methods for detection of Metallo- $\beta$ lactamase producing $P$. aeruginosa isolates. J.Cell Tissue Research. 2011; 11(1):2601-
2604.

Queenan AM, Bush K. Carbapenemases: the Versatile b-lactamases. Clin Microbiol Rev. 2007;20:440-58, doi: 10.1128/CMR.0000107.

Sader HS, Reis AO, Silbert S, Gales AC. IMPs, VIMs and SPMs: the diversity of metallo-blactamases produced by carbapenemresistant Pseudomonas aeruginosa in a Brazilian hospital. Clin Microbiol Infect. 2005;11:73-6, doi: 10.1111/j.14690691.2004.01031.x.

Shobha KL, Lenka PR, Sharma MK, Ramachandara L, Bairy I. Metallo- $\beta$ lactamase production among Pseudomonas species and Acinetobacter species in costal Karnataka. J Clin and Diagnostic Research. 2009;76(5):1747-1753.

Tavajjohi Z, Moniri R and Khorshidi A. Detection and characterization of multidrug resistance and extended-spectrum-beta-lactamaseproducing (ESBLS) Pseudomonas aeruginosa isolates in teaching hospital. African Journal of Microbiology Research. 2011; 5(20):3223-3228.

Upadhyay S, Sen MR, Bhattacharjee A. Presence of different beta-lactamase classes among clinical isolates of Pseudomonas aeruginosa expressing AmpC betalactamase enzyme. J Infect Dev Ctries. 2010;4(4):239-42.

Yong D, Lee K, Yum JH, Shin HB, Rossolini GM, Chong Y. Imipenem-EDTA disk method for differentiation of metallo- $\beta$ lactamases producing clinical isolates of Pseudomonas spp. and Acinetobacter spp. J Clin Microbiol.2002;40(4):3798-801.

\section{How to cite this article:}

Soumya S. and Mahantesh B. Nagmoti. 2017. Comparison of Different Phenotypic Tests for the Detection of Metallo-B-Lactamase (MBL) Producing Pseudomonas aeruginosa. Int.J.Curr.Microbiol.App.Sci. 6(10): 3231-3238. doi: https://doi.org/10.20546/ijcmas.2017.610.378 